\title{
DOUBLE TRANSITIVITY IN FINITE PROJEGTIVE PLANES
}

\author{
T. G OSTROM
}

Introduction. A projective plane is characterized to a certain extent by the amount of transitivity it possesses. This amounts essentially to saying that the plane is characterized by its group of collineations. The transitive planes that have been most thoroughly studied are the cyclic planes $(8 ; 10$; $12 ; 13 ; 14) .{ }^{1}$ It is believed that all finite cyclic planes are Desarguesian, but it has not been proved that this is the case. Infinite cyclic non-Desarguesian planes are known to exist (10). Closely allied are cyclic affine planes of Hoffman (11). Zappa (17) has extended some of the notions of cyclic planes to the case where the plane is transitive under a group of collineations which is not necessarily cyclic. He arrives at a representation of the plane essentially the same as Bruck's group difference sets (6). Baer (3) has used a limited kind of transitivity to coordinate planes. Bruck and Kleinfeld have shown that alternative planes are transitive on quadrangles (7).

In this paper, we are limited to finite planes with $n+1$ points on a line, where $n$ is odd and not a square. We show that such planes, if doubly transitive, are Desarguesian. If they are doubly transitive on points not on a special line, they are Veblen-Wedderburn planes which cannot be coordinatized by a near-field.

\section{Definitions and preliminary lemmas}

Definition: A plane $\pi$ will be said to be doubly transitive if, for every two ordered pairs of points $A, B$, and $C, D$ (where $A \neq B$ and $C \neq D$ ) there is a collineation of $\pi$ such that $C$ is the image of $A$ and $D$ is the image of $B$.

Lemma 1. If a collineation $\sigma$ leaves fixed every point on a line $l$ and every line through a point $P$, then no point not on $l$ (with the possible exception of $P$ ) is fixed unless $\sigma$ is the identity.

Proof. Suppose that $Q \notin l$ is fixed by $\sigma$, where $Q \neq P$. Since every line through $Q$ and a point of $l$ is fixed, every line through $Q$ is fixed. Then every point in the plane is fixed, since it must lie on a line through $Q$ and a line through $P$. Points on the line $P Q$ are exceptions, but they must be fixed if every other point of the plane is fixed.

Received November 15, 1955

${ }^{1}$ There is a misstatement in (14). On page 422 , the remark immediately preceding Theorem 2.5 should read ". . . if two ovals have more than half of their points in common ...." We should also remark that the term "perspectivity" as used in that paper is not intended to indicate a collineation. 
Remark. It is fairly well known that if a collineation fixes every point on a line, then it fixes every line through some point.

Definition: A collineation (not equal to the identity) which leaves fixed every point on a line $l$ and every line through a point $P$ will be called a perspectivity. The line $l$ will be called the axis of the perspectivity and $P$ will be called the center.

Definition: An involution is a collineation of order two.

Lemмa 2. If $n$ is odd and not a square, every involution is a perspectivity in which the center does not lie on the axis.

Proof. A collineation of order two leaves fixed the point of intersection of each line with its image. Likewise, a line through a point and its image will be fixed. The intersection of two fixed lines is a fixed point. Thus every fixed line contains a fixed point and we have already shown that every other line contains a fixed point. Our collineation $\sigma$ is what Baer (4) calls a quasiperspectivity. He has shown that every quasi-perspectivity is either a perspectivity or leaves fixed a subplane of order $n^{\frac{1}{2}}$. Since $n$ is not a square, $\sigma$ is a perspectivity. If the center $P$ lies on the axis, consider any line (not equal to the axis) which goes through $P$. By Lemma 1, we have a fixed line on which $P$ is the only fixed point. The $n$ non-fixed points are interchanged in pairs, and $n$ must be even.

LEMma 3. If the plane $\pi$ is doubly transitive, $\pi$ admits at least one involution.

Proof. Let $A$ and $B$ be any two points of $\pi$. If $\pi$ is doubly transitive, there exists a collineation $\sigma$ such that $A \rightleftharpoons B$. Thus $\sigma$ is of even order and, since $\pi$ is finite, some power of $\sigma$ is of order two.

LEMMA 4. Let $\rho$ and $\sigma$ be two involutions with the same axis $l$ but with different centers. Then $\rho \sigma$ is a perspectivity with axis $l$ and center on $l$.

Proof. Since every point on $l$ is left fixed by both $\rho$ and $\sigma$, these points are left fixed by $\rho \sigma$. Suppose that some point $Q$ not on $l$ is fixed by $\rho \sigma$. If $\rho$ interchanges $Q$ with some point $Q_{1}$, then $\sigma$ must do likewise and therefore both $Q$ and $Q_{1}$ are fixed by $\rho \sigma$. If $Q=Q_{1}$, then $Q$ must be the common center for $\rho$ and $\sigma$. If $Q \neq Q_{1}, \rho \sigma$ is the identity by Lemma 1 and $\rho=\sigma$.

Lemma 5. Let $\rho$ and $\sigma$ be two involutions with the same center $P$ but with different axes. Then $\rho \sigma$ is a perspectivity with center $P$ and axis $l$ which goes through $P$.

LEMMA 6. Let $\rho$ and $\sigma$ be two involutions such that the axis of each is incident with the center of the other. Then $\rho \sigma$ is an involution with axis going through the centers of $\rho$ and $\sigma$ and center on the intersection of the axes of $\rho$ and $\sigma$. 
Proof. Let $\rho$ have center $P$ and axis $l$. Let $Q$ and $m$ be the center and axis respectively of $\sigma$. Let $l$ and $m$ intersect in $R$. Then $P \in m$ and $Q \in l$. $P, Q$, and $R$ are fixed by both $\rho$ and $\sigma$, and therefore by $\rho \sigma$. Likewise, lines $l, m$, and $P Q$ are fixed by both $\rho$ and $\sigma$. Let $A$ and $A_{1}$ be two points on $l$ interchanged by $\sigma$. Since all points of $l$ are fixed by $\rho, \rho \sigma$ also interchanges $A$ and $A_{1}$. Similarly, if $\rho$ interchanges $B$ and $B_{1}$ on $m, \rho \sigma$ also interchanges $B$ and $B_{1}$. Thus, except for $P, Q$, and $R$, the points on $l$ and $m$ are interchanged in pairs by $\rho \sigma$.

Let $X$ be any point not on the sides of the triangle $P Q R$. Let $P X$ intersect $l$ in the point $A$ and let $Q X$ intersect $m$ in the point $B$. Let $A \rightleftharpoons A_{1}$ and $B \rightleftharpoons B_{1}$ under $\rho \sigma$. Then if $P A_{1}$ and $Q B_{1}$ intersect in $X_{1}, X$ and $X_{1}$ are interchanged by $\rho \sigma$. We conclude that $\rho \sigma$ must be an involution with axis $P Q$ and center $R$.

Definition. If there is an involution with center $P$ and axis $l, l$ will be said to be an axis of $P$.

\section{Doubly transitive planes}

Theorem 1. Let $\pi$ be a doubly transitive finite plane, where $n$ is odd and not a square. Then $\pi$ is Desarguesian.

Proof. By Lemmas 2 and $3, \pi$ admits an involution $\sigma$ with some axis $l$ and center $P \notin l$. Let $A$ be some point on $l$ and $A_{1}$ be some point not on $l$. Consider the collineation $\rho$ which carries $P$ into $P$ and $A$ into $A_{1}$. This collineation will carry $l$ into some line $l_{1} \neq l$. Then $l_{1}$ will be an axis of $P$ with respect to the involution $\rho^{-1} \sigma \rho$.

Let $l$ and $l_{1}$ intersect in $Q$. Then $Q$ lies on at least two axes of $P$. Suppose that $Q$ lies on exactly $k$ axes of $P$. Given any point $Q_{1} \neq P$, there is a collineation which leaves $P$ fixed and carries $Q$ into $Q_{1}$. Hence every point except $P$ lies on $k$ axes of $P$, since a collineation which fixes $P$ maps axes of $P$ into axes of $P$.

Now no line through $P$ can be an axis of $P$, since $n$ is odd. Consider any line through $P$. This line will contain $n$ points besides $P$, each lying on $k$ axes of $P$. Hence there are exactly $n k$ axes of $P$.

If $l$ is some axis of $P$, each of the $n+1$ points on $l$ must lie on $k-1$ axes of $P$ besides $l$ itself. Hence

$$
n k=(n+1)(k-1)+1
$$

and $k=n$. Therefore, each of the $n^{2}$ lines not through $P$ is an axis of $P$.

Now, let $Q \neq P$ and let $\sigma=\sigma_{1}, \sigma_{2}, \ldots, \sigma_{n}$ be involutions corresponding to the $n$ axes of $P$ which go through $Q$. Then $1=\sigma \sigma_{1}, \sigma \sigma_{2}, \ldots, \sigma \sigma_{n}$ are $n$ distinct collineations, which by Lemma 5 are perspectivities with center $P$ and axes which go through $P$. Moreover, they all have the same axis $P Q$, since $Q$ is fixed in each case. Let $A$ be any point not on the line $P Q$. The images of $A$ under the collineations $\tau_{i}=\sigma \sigma_{i}$ all lie on the line $A P$. Moreover, if $\tau_{i} \neq \tau_{j}$, the image of $A$ under $\tau_{i}$ is different from its image under $\tau_{j}$ for otherwise $\tau_{i} \tau_{j}{ }^{-1}$ would leave $A$ fixed as well as every point on $P Q$ and every 
line through $P$. By Lemma $1, \tau_{i} \tau_{j}^{-1}$ would then be the identity. Since there are $n$ collineations $\tau_{i}, A$ can be carried into every point on $A P$ except $P$ by some collineation $\tau_{i}$.

Baer (3, Theorem 6.2) has shown that this implies that the Minor Theorem of Desargues is satisfied for center $P$ and axis $P Q$. By further use of double transitivity, there are sufficient collineations to leave $P$ fixed and carry $P Q$ into any line through $P$, and finally $P$ can be taken into any point. Thus the Minor Theorem of Desargues is satisfied throughout and the plane is alternative (9). But Zorn (18) has shown that every finite alternative division ring is Desarguesian.

\section{Planes doubly transitive except for points on a special line}

Lemma 7. Let the plane $\pi$ admit a group of collineations $\Sigma$ which is doubly transitive for points not on the line $l_{\infty}$. Then (a) if $P \in l_{\infty}$, the group which leaves $P$ and $l_{\infty}$ fixed is transitive on finite points and (b) $\Sigma$ is transitive over points on $l_{\infty}$.

Proof. Without loss of generality, we can assume that $\Sigma$ leaves $l_{\infty}$ fixed, since otherwise $\pi$ is doubly transitive on all points and the plane is Desarguesian. Let $A, B$ and $A_{1}, B_{1}$ be two pairs of finite points such that $A, B$ and $P$ are collinear and also $A_{1}, B_{1}$, and $Q$ are collinear, where $Q \in l_{\infty}$. Then the member of $\Sigma$ which carries the pair $A, B$ into the pair $A_{1}, B_{1}$ will carry $P$ into $Q$. If $P=Q, P$ is fixed.

Definition. A perspectivity with axis $l_{\infty}$ and center on $l_{\infty}$ will be called a translation.

Lemma 8. Let $\pi$ be a plane of order $n$, where $n$ is odd and not a square. If $\pi$ admits a group $\Sigma$ of collineations as in Lemma 7 , then $\pi$ admits a translation.

Proof. As in Lemmas 2 and 3, $\pi$ admits an involution with some axis $l$ and center $P \notin 1$. Suppose first that $l_{\infty}$ is the axis of an involution and $P \notin l_{\infty}$ is the center. Since we have transitivity on finite points, there is another involution with center $P_{1} \neq P$, and with $l_{\infty}$ as axis. In this case, Lemma 8 follows from Lemma 4.

On the other hand, suppose that there is an involution with some ordinary line $l$ as axis. Since $l_{\infty}$ is fixed, the center $P$ is on $l_{\infty}$. From Lemma 7 (a), every finite point is on an axis of $P$ and $P$ has more than one axis.

Now if two axes of $P$ go through the same point $Q \in l_{\infty}$ it follows from Lemma 5 that there is a perspectivity with center $P$ and axis through $P$. Moreover, $Q$ is on the axis (by Lemma 1) which must therefore be $l_{\infty}$.

Thus, Lemma 8 is established except for the possibility that no two axes of $P$ intersect in a point on $l_{\infty}$. Let $m$ be an ordinary line through $P$. Corresponding to the $n$ finite points on $m$, there are $n$ axes of $P$. If no two of them intersect on a point of $l_{\infty}$, every point on $l_{\infty}$ except $P$ must lie on an axis of $P$. From Lemma 7 (b), it follows that a similar statement must hold for every 
point on $l_{\infty}$. Thus, there exist points $P$ and $Q \in l_{\infty}$ for which the conditions of Lemma 6 apply and $l_{\infty}$ is the axis of some involution. But we have already shown that Lemma 8 follows in this case.

THEOREM 2. Let $\pi$ be a plane of order $n$, where $n$ is odd and not a square and let $\pi$ be doubly transitive on points not belonging to the line $l_{\infty}$. Then (a) $\pi$ is a Veblen-Wedderburn plane and (b) $\pi$ is Desarguesian if any of the coordinate rings (using $l_{\infty}$ as the special line) satisfy the left distributive law or the associative law for multiplication.

Proof. By Lemma 8, $\pi$ admits a translation. Suppose that $A \rightarrow B$ under a translation. From the double transitivity, it follows that any finite point $A_{1}$ can be carried into any finite point $B_{1}$ by a translation. (Recall that, without loss of generality, we can assume that $l_{\infty}$ is fixed by all collineations.) Thus finite points are transitive under translations. André (1) calls such planes translations planes and has shown that they are Veblen-Wedderburn planes.

André also has divided translations planes into six mutually exclusive classes (2, Theorem 4). In cases II and IV, the plane is not transitive on infinite points, contrary to Lemma 7. Case III is Desarguesian if $\pi$ is finite. Case $\mathrm{V}$ is doubly transitive on finite points, but $n$ is a square $(n=9)$. The remaining two cases are those in which $\pi$ is Desarguesian or in which no coordinate ring satisfies either the left distributive law or the associative law.

\section{REFERENCES}

1. J André, Ueber nicht-Desarguesche Ebenen mit transitiver Translationsgruppe, Math. Z. 60 (1954), 156-186.

2. - Projektive Ebenen über Fastkörpern, Math. Z. 62 (1955), 137-160.

3. R. Baer, Homogeneity of projective planes, Amer. J. Math. 64 (1942), 137-152.

4. - Projectivities with fixed points on every line in the plane, Bull. Amer. Math. Soc. 52 (1946), 273-286.

5. - - Projectivities of finite projective planes, Amer. J. Math. 69 (1947), 653-684

6. R H. Bruck, Difference sets in a finite group, Trans. Amer. Math. Soc. 78 (1955), 464-481.

7. - and E. Kleinfeld, The structure of alternative division rings, Proc. Amer. Math. Soc. 2 (1951), 878-890.

8. T. A. Evans and H. B. Mann, On simple difference sets, Sankhya 11 (1951), 357-364.

9. M. Hall, Projective planes, Trans. Amer. Math. Soc. 54 (1943), 229-277.

10. - Cyclic projective planes, Duke Math. J. 14 (1947), 1079-1090.

11. A. J. Hoffman, Cyclic affine planes, Can. J. Math. 4 (1952), 295-301.

12. H B Mann, Some theorems on difference sets, Can. J. Math. 4 (1952), 222-226.

13. T. G. Ostrom, Concerning difference sets, Can. J. Math. 5 (1953), 421-424.

14. - Ovals, dualities, and Desargues's theorem, Can. J. Math. 7 (1955), 417-431.

15. J. Singer, A theorem in finite projective geometry and some applications to number theory, Trans. Amer. Math. Soc. 48 (1938), 377-385.

16. L. Skornyakov, Projective planes, Translation No. 99, Amer. Math. Soc. (1951).

17. G. Zappa, Sui piani grafica finiti transitivi e quasi-transitivi, Ric. di Mat., II (1953), 274-287.

18. M. Zorn, Theorie der alternativen Ringe, Abh. Math. Sem. Hamb. Univ. 8 (1930), 123-147.

Montana State University 